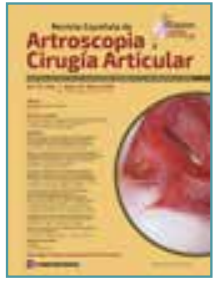

Vol. 25. Fasc. 1. Núm. 61. Marzo 2018

ISSN: 2386-3129 (impreso)

2443-9754 (online)

\title{
Originales
}

\section{Resultados objetivos de la ligamentoplastia de ligamento cruzado anterior en la edad pediátrica mediante la técnica intraepifisaria (all-epiphyseal)}

\author{
Z. González'1, J. Duart'², A. Valentî́ ${ }^{3}$ N. Urquiza', AD. Cuéllar', \\ L. Montes ${ }^{1}$, S. García², AS. Cuéllar'1, R. Cuéllar ${ }^{1}$
}

\author{
${ }^{1}$ Hospital Universitario de Donostia. Guipuzkoa \\ ${ }^{2}$ Complejo Hospitalario de Navarra. Pamplona \\ ${ }^{3}$ Clínica Universidad de Navarra. Pamplona
}

Correspondencia:

Dra. Zaira González Fernández

Correo electrónico: zgfernandez@gmail.com
Recibido el 2 de septiembre de 2017

Aceptado el 4 de marzo de 2018

Disponible en Internet: marzo de 2018

\section{RESUMEN}

Objetivo: la rotura del ligamento cruzado anterior (LCA) en edad pediátrica es una lesión cuya incidencia tiene una tendencia creciente en los últimos años. El diagnóstico se basa en la exploración física complementada con pruebas radiológicas. La actitud terapéutica actual es cada vez más frecuente que sea quirúrgica debido a las lesiones articulares, presentes o potenciales, secundarias a la inestabilidad y a su alto nivel de actividad a pesar de la lesión del LCA. La técnica más elegida es la técnica all-epiphyseal. El objetivo es revisar la casuística y los resultados de los pacientes intervenidos mediante esta técnica en una cohorte procedente de 3 centros diferentes.

Método: se analiza una serie de casos de roturas de LCA en edad pediátrica intervenidas mediante ligamentoplastia mediante técnica all-epiphyseal, en el Hospital Universitario de Donostia, el Complejo Hospitalario de Navarra y la Clínica Universidad de Navarra, y su comparación con los resultados bibliográficos revisados.

Resultados: se presentan 18 casos, entre 9 y 15 años, tratados entre 2014 y 2016. Las lesiones asociadas estaban presentes en 14 de los casos; la más frecuente fue la rotura

\begin{abstract}
Objective results of anterior cruciate ligament ligamentoplasty in pediatric age using "all-epiphyseal" technique
\end{abstract}

Aim of study: anterior cruciate ligament (ACL) tears in pediatric patients is an injury which incidence is currently increasing. The base of the diagnosis is physical exploration complemented with $X$-ray and magnetic resonance imaging (MRI). The actual treatment line is frequently to perform a surgery due to articular injuries, at risk or already existing, produced by knee instability and children's high level activity despite of ACL injury. The most used surgical technique is "all-epiphyseal". The aim of this study is to examine casuistry and results of patients surgically treated with this technique in 3 different hospitals. Method: pathogenesis and results of pediatric ACL tears case series surgically treated with "all-epiphyseal" technique are shown. Patients come from Hospital Universitario de Donostia, Complejo Hospitalario de Navarra and Clínica Universidad de Navarra and results are compared with the ones previously published and reviewed.
FS https://doi.org/10.24129/j.reaca.25161.fs1709030

(c) 2018 Fundación Española de Artroscopia. Publicado por Imaidea Interactiva en FONDOSCIENCE ${ }^{\circledR}$ (www.fondoscience.com). Este es un artículo Open Access bajo la licencia CC BY-NC-ND (www.creativecommons.org/licenses/by-nc-nd/4.0/). 
del menisco interno (MI) (8 casos). Hubo complicaciones postoperatorias en 2 de los casos. La reinserción deportiva se realizó entre los 4 y los 14 meses, tiempo en el cual 11 pacientes tuvieron algún tipo de consecuencia social por ello (aislamiento ocasional o peor rendimiento académico). El resultado final a 1 año de evolución es el 100\% de los pacientes sin dolor, balance articular (BA) completo en 15 casos y 3 con leve limitación y 2 pacientes con sensación de inestabilidad. La laxitud se cuantificó mediante KT1000, con 15 pacientes sin diferencias y 3 con diferencia kt30 de 2 . Conclusiones: la ligamentoplastia mediante la técnica intraepifisaria en pacientes Tanner I-III previene el avance o la aparición de otras lesiones intraarticulares, obtiene buenos resultados funcionales, una reinserción deportiva completa y soluciona los problemas sociales secundarios pero importantes como el aislamiento de su entorno habitual o las consecuencias académicas.

Nivel de evidencia: nivel III.

Relevancia clínica: este estudio intenta avanzar en la evidencia sobre la necesidad del tratamiento de lesiones deportivas en la edad pediátrica, una patología reciente y en aumento.

Palabras clave: Rotura LCA. All-epiphyseal. Artroscopia infantil. Rodilla pediátrica. Fisis.

\section{Introducción}

La rotura del ligamento cruzado anterior (LCA) en la población pediátrica supone menos del $5 \%$ de las lesiones totales de LCA, el 30\% de las lesiones de rodilla en los adolescentes futbolistas. Aunque el rango de edad de presentación es relativamente estrecho, muy raras por debajo de los 9 años de edad ${ }^{(1,2)}$, el tratamiento quirúrgico ha aumentado de 17,6 reconstrucciones cada 100.000 personas en 1990 a 50,9 en 2009 en los EE. UU. ${ }^{(3,4)}$.

El mecanismo de lesión es similar que en los adultos: un traumatismo indirecto en el $80 \%$ de los casos o situaciones de no contacto cuando se combina deceleración y movimientos de rotación pivotando sobre la articulación $n^{(2)}$. El diagnóstico se basa en la anamnesis, la clínica y la exploración física comparada con la rodilla contralateral y pruebas radiológicas complementarias: radiología simple y resonancia magnética (RM). A edades tempranas, la RM tiene gran sensibilidad y especificidad para las lesiones de LCA, pero también
Results: there are 18 patients, 9 to 15 years old, treated from 2014 to 2016. Concomitant articular injuries were present in 14 of these cases, and the most frequent was internal meniscus tear (8 cases). Only 2 had post operatory complications. Return to sports was from 4 to 14 months postoperatory. In this period 11 patients had any kind of social impairment because lack of participation in the activity (social isolation or lower academic marks). Final results after 1 year in 100\% cases with no pain, complete ROM in 15 cases and 3 with minor limitation. Two patients reported lasting instability. Laxity was KT1000 assessed: 15 patients had no difference and 3 has a difference of 2 in kt30.

Conclusions: $\mathrm{ACL}$ repair by using all-epiphyseal technique in patients Tanner I-III prevents worsening or new articular injuries, has good functional results, a complete reintegration to sports and gives solution to secondary but important social consequences as isolation or altering academic results.

Level of evidence: III.

Clinical relevance: this study tries to improve the actual evidence about the need of treatment of sports injuries in pediatric orthopedics because is recently assessed and it's incidence is increasing.

Key words: ACL tear. All-epiphyseal. Pediatric arthroscopy. Children knee. Physis.

alta tasa de falsos positivos para lesiones meniscales $^{(5)}$. Aun así, la exploración física es básica. Existe un $78 \%$ de concordancia entre los hallazgos clínicos y artroscópicos, un 78\% de desacuerdo entre artroscopia y RM, y un 75\% de desacuerdo entre clínica y $\mathrm{RM}^{(4)}$. Están descritas lesiones asociadas hasta en la mitad de los pacientes ${ }^{(1,5)}$ y puede haber lesiones asociadas en el ligamento colateral medial, meniscos o la esquina posterolateral ${ }^{(6)}$.

Las consecuencias de la rotura del LCA no son solo biomecánicas y anatómicas. Los niños forman parte de círculos sociales que se mueven en torno a su actividad principal que, en este caso, es el deporte. En este círculo social también están incluidas de forma activa la propia familia, las amistades del niño y de sus padres y, por lo tanto, la gran mayoría de las actividades fuera del ámbito escolar. Estos niños, al detener su actividad deportiva, quedan indudablemente excluidos en alguna medida de parte de su vida escolar, su vida extraescolar y sus amistades habituales. En 
el periodo inicial puede asistir a los eventos pero, si la actividad se detiene, puede ocurrir la exclusión en muchos casos. Esto tiene 2 consecuencias: repercusión en el resto de los aspectos de su vida diaria (escolar o familiar) y contribuye o es la causa de que, a pesar de la lesión, no sigan las recomendaciones para realizar un tratamiento conservador y que lleva a un aumento en las indicaciones de tratamiento quirúrgico en las lesiones de LCA pediátrico.

En este contexto, la evolución de la abstinencia terapéutica no está del todo clara(7). Los niños siguen siendo activos a pesar de la lesión, no adaptan como los adultos su actividad y aumenta el riesgo de más lesiones intraarticulares por episodios recurrentes de inestabilidad ${ }^{(4)}$. Otra consecuencia es que las indicaciones de tratamiento conservador son escasas: roturas incompletas sin inestabilidad ni lesiones asociadas, con menos de un 50\% de afectación del espesor del ligamento, menos de 14 años y pivot y Lachman similar a la rodilla contralateral ${ }^{(1,6)}$. Aun así, el $50-65 \%$ de los pacientes presentan lesiones concomitantes en el momento del diagnóstico o en un momento posterior, por lo que la tendencia es al tratamiento quirúrgico ${ }^{(1,6)}$.

El tratamiento quirúrgico se inicia obligatoriamente con una exploración artroscópica completa en busca de lesiones desapercibidas y la realización de una ligamentoplastia con autoinjerto de semitendinoso y/o recto interno $(\mathrm{ST} / \mathrm{RI})^{(1,6)}$, tendón cuadricipital o aloinjerto(7).

La técnica difiere según la edad esquelética con el fin de minimizar las alteraciones del crecimiento ${ }^{(3,6,8)}$, una complicación severa, probablemente infradiagnosticada ${ }^{(9)}$ y de la cual hay que informar correctamente a los padres ${ }^{(6)}$. Las técnicas que se utilizan con más frecuencia son: plastia con banda iliotibial para Tanner I-II, técnica all-epiphyseal o intraepifisaria de Anderson (TIE) para Tanner I-III y técnica de transfixión fisaria para pacientes con mayor edad ósea (Tanner IV-V $)^{(5,7)}$. La tendencia es a utilizar técnicas TIE en pacientes esqueléticamente inmaduros en general (Tanner I-III) $)^{(3)}$ porque las técnicas con fascia iliotibial tienen peores resultados ${ }^{(7)}$.

Es necesario que el protocolo postoperatorio se lleve a cabo por un equipo rehabilitador infantil. Los niños tienen menor implicación en un proceso que en ellos es de por sí más lento(3). Habitualmente se mantiene una ortesis en exten- sión hasta 6 semanas y apoyo-contacto durante 4 semanas, e inicio de ejercicios dirigidos desde la segunda semana hasta la reinserción deportiva total alrededor del año tras la cirugía(2,5).

En este estudio se pretende revisar la casuística y los resultados de las ligamentoplastias de LCA en la población pediátrica atendida en 3 centros diferentes (Hospital Universitario de Donostia, Completo Hospitalario de Navarra y Clínica Universidad de Navarra) mediante TIE y analizar la evolución y los resultados clínicos, las complicaciones y la repercusión social con la premisa de que es una técnica necesaria.

\section{Métodos}

Se realiza la revisión retrospectiva de una serie de casos detectados e intervenidos entre 2014 y 2016 en el Hospital Universitario de Donostia, el Complejo Hospitalario de Navarra y la Clínica Universidad de Navarra en la población pediátrica (hasta 15 años de edad). Los criterios de inclusión han sido los criterios quirúrgicos para la lesión de LCA pediátrico: diagnóstico clínico y radiológico de rotura completa o parcial de LCA con lesiones asociadas, rotura completa de LCA aislada y

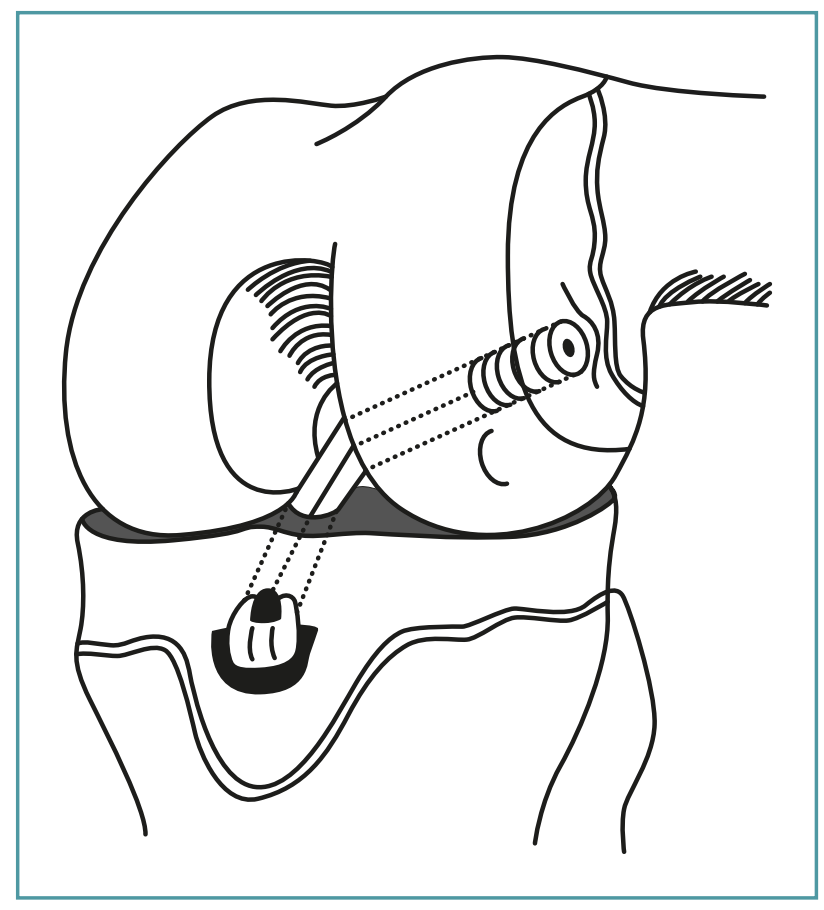

Figura 1. Ligamentoplastia all-epiphyseal según sistema de OrthoPediatrics ${ }^{\circledR}$. 

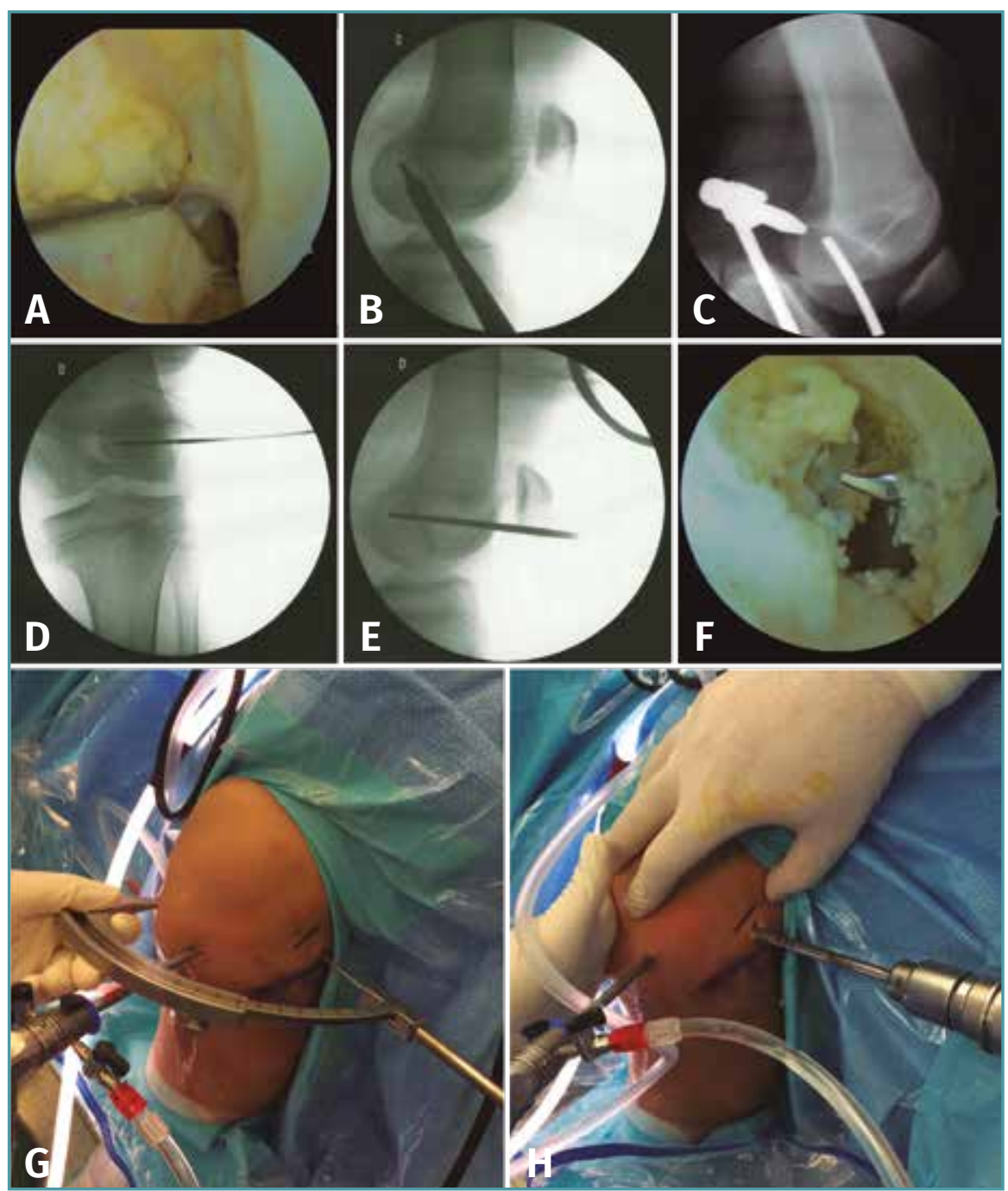

Figura 2. Túnel femoral. A: inserción femoral del ligamento cruzado anterior (LCA) desnuda; B: punto de inserción de la guía femoral, tomando como referencia la cortical posterior y la línea de Bloomensat; C: Posición de la guía en la escopia; $D$ y E: posición de la guía femoral en la epífisis distal; F: guía femoral en la huella del $L C A ; G$ y F: vista externa de la colocación de la guía y brocado epifisario femoral.

apoyado por artroscopistas de su servicio en el Complejo Hospitalario de Navarra (7 casos) y un cirujano ortopédico especialista en rodilla en la Clínica Universidad de Navarra (3 casos).

La técnica quirúrgica utilizada por los 3 centros ha sido la ligamentoplastia mediante sistema all-epiphyseal de OrthoPediatrics ${ }^{\circledR}$. Se trata de una plastia retrógrada guiada por escopia intraoperatoria (Figura 1). El punto de inicio es la realización de un túnel horizontal desde la cara lateral del cóndilo femoral hasta la huella del LCA en el intercóndilo con la guía femoral a $60^{\circ}$, como se muestra en la Figura 2, y un túnel tibial con guía a $30^{\circ}$ iniciando la entrada desde un punto medial a la tuberosidad tibial anterior (TTA) hasta la inserción tibial del LCA (Figura 3). Es recomendable verificar con el artroscopio que el recorrido del túnel no atraviesa la fisis (Figura 4). En la tibia el trayecto es más horizontal que en las técnicas de transfixión fisaria, con el fin de evitar dañar la fisis que, en la parte anterior de la tibia, desciende hasta la TTA.

roturas parciales con inestabilidad articular. Las pruebas diagnósticas realizadas han sido: exploración clínica (balance articular-BA-, maniobras meniscales, test de Lachman, test de pivot shift y resto de pruebas de estabilidad articular en busca de lesiones asociadas) y radiológicas (radiología simple y RM).

La cirugía ha corrido a cargo de un cirujano ortopédico infantil con experiencia en artroscopia junto con un cirujano artroscopista en el Hospital Universitario de Donostia (8 casos), un cirujano ortopédico infantil con formación en artroscopia
Se introduce un injerto simple de ST o RI desde el túnel femoral hasta un anclaje en tibia (Figura 5) y se fija con un torillo reabsorbible en el túnel femoral (Figura 6). El resultado final se muestra en la Figura 7.

Los puntos importantes a la hora de realizar TIE son los siguientes:

1. Brocado a bajas revoluciones (ream en vez de drill) para minimizar el riesgo de necrosis térmica de fisis por proximidad.

2. Tensionar la plastia con la rodilla a un máximo de $45^{\circ}$ de flexión y no realizar maniobras 


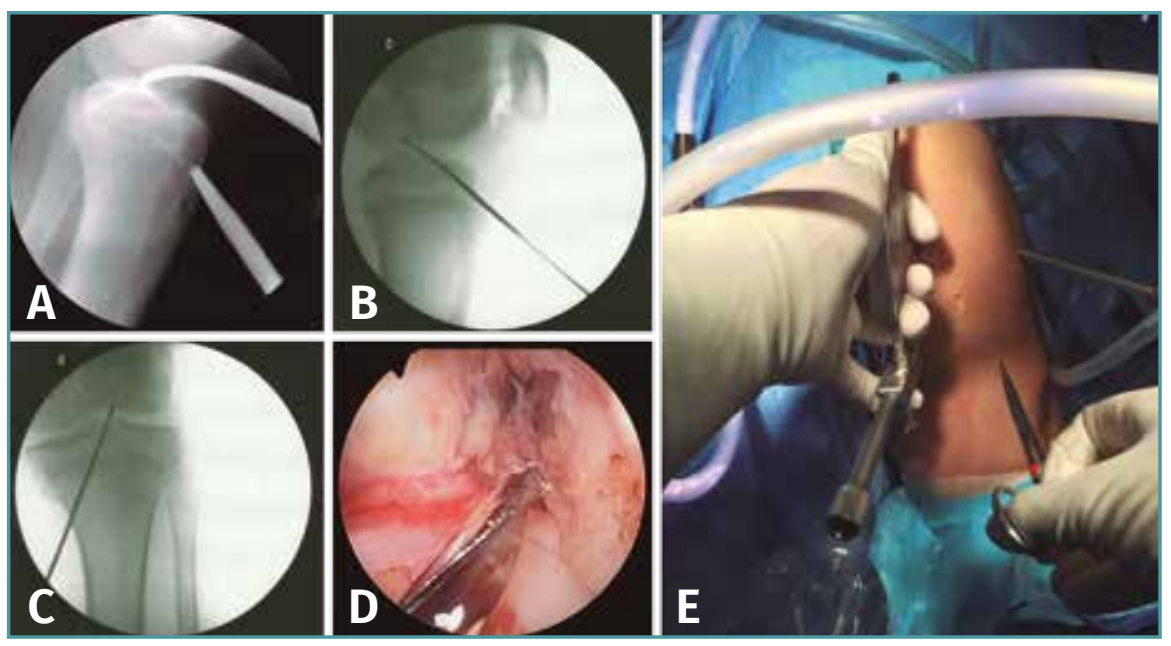

Figura 3. Túnel tibial. A: posición de la guía femoral en la escopia; B y C: posición de la guía epifisaria tibial; $D$ : salida de la guía tibial en la inserción distal del LCA; E: vista externa del tiempo tibial.

de reducción de cajón anterior para evitar una "hiperreducción" secundaria a la elasticidad fisiológica infantil que resulta en un déficit de extensión.

3. No aplicar excesiva tensión sobre el injerto. Puede producir alteraciones de las fisis locales.

4. Los túneles han de ser del menor diámetro posible y con la mínima oblicuidad con el fin de dañar la menor área articular.

5. Realizar limpieza intercondílea y trocleoplastias moderadas con uso mínimo de vaporiza-

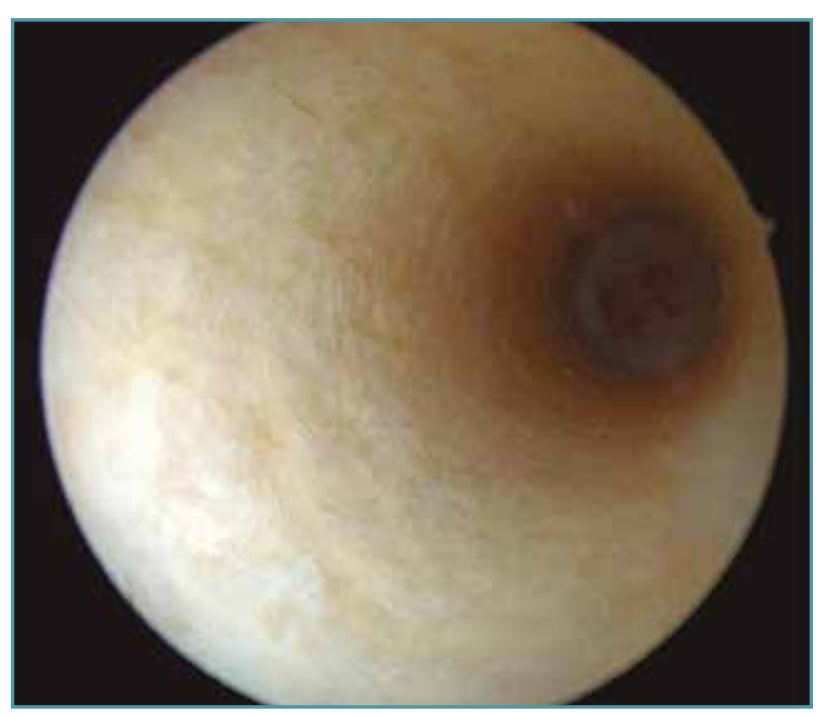

Figura 4. Vista del túnel epifisario. No se observa zona fisaria en su recorrido. dor por riesgo de lesión del anillo pericondral de la fisis por su proximidad.

La elección del tipo de injerto ha sido a favor del aloinjerto en el Hospital Universitario de Donostia y de autoinjerto (isquiotibiales ipsilaterales) en el Complejo Hospitalario de Navarra y la Clínica Universidad de Navarra.

Siempre, previa a la ligamentoplastia, se ha hecho una exploración artroscópica completa y se han documentado lesiones existentes y se han reparado si ha sido posible. El protocolo postoperatorio ha sido el habitual ${ }^{(6)}$ y se ha llevado a cabo en colaboración con un equipo rehabilitador infantil. Los niños tienen menor implicación en un proceso que en ellos es de por sí más lento(3). Habitualmente, se mantiene una ortesis en extensión hasta 6 semanas y apoyo-contacto durante 4 semanas e inicio de ejercicios dirigidos desde la segunda semana hasta la reinserción deportiva total entre 7 y 12 meses tras la cirugia $(2,6,10)$.

Las revisiones por el equipo quirúrgico han sido periódicas con el fin de descartar lesiones neurovasculares, infecciones, problemas con el material, artrofibrosis y cualquier tipo de complicación, además de buscar la recuperación precoz del BA. En la última revisión, al año de la cirugía, se ha recogido la dif30 en el KT1000 entre la rodilla sana y la intervenida, el BA final, el estado general y los datos de repercusión social de la lesión. Las pruebas radiológicas complementarias (radiología simple o RM) se han reservado a los casos con evolución no favorable.

\section{Resultados}

La cohorte está compuesta por 18 pacientes intervenidos mediante TIE: 5 niñas y 13 niños con edades comprendidas entre los 9 y los 15 años (media: 12 años) (Tabla 1).

El mecanismo de lesión más frecuente ha sido el traumatismo deportivo con un total de 15 ca- 

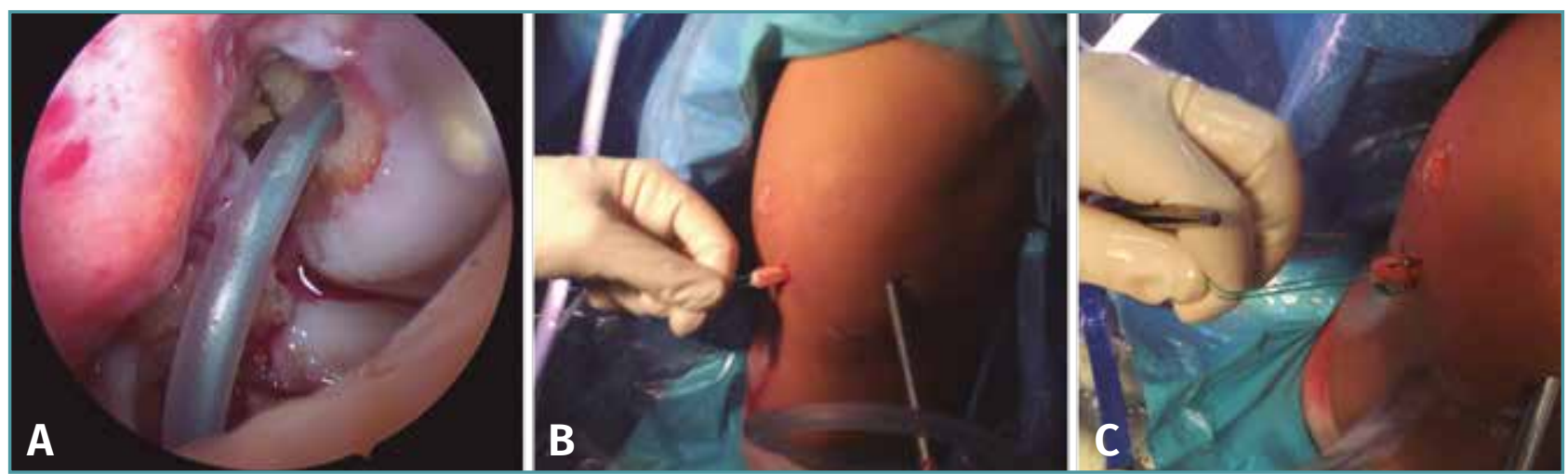

Figura 5. Inserción de la plastia. A: pasador para guiar la plastia desde el fémur a la tibia; B y C: extracción del bucle distal y colocación del tope tibial (ArmorLink ${ }^{\circledR}$ ).

sos, de los cuales 11 han sido jugando al fútbol, 2 realizando esquí, 1 jugando al baloncesto y 1 jugando al rugby. Los restantes han sido caídas casuales (Tabla 1).
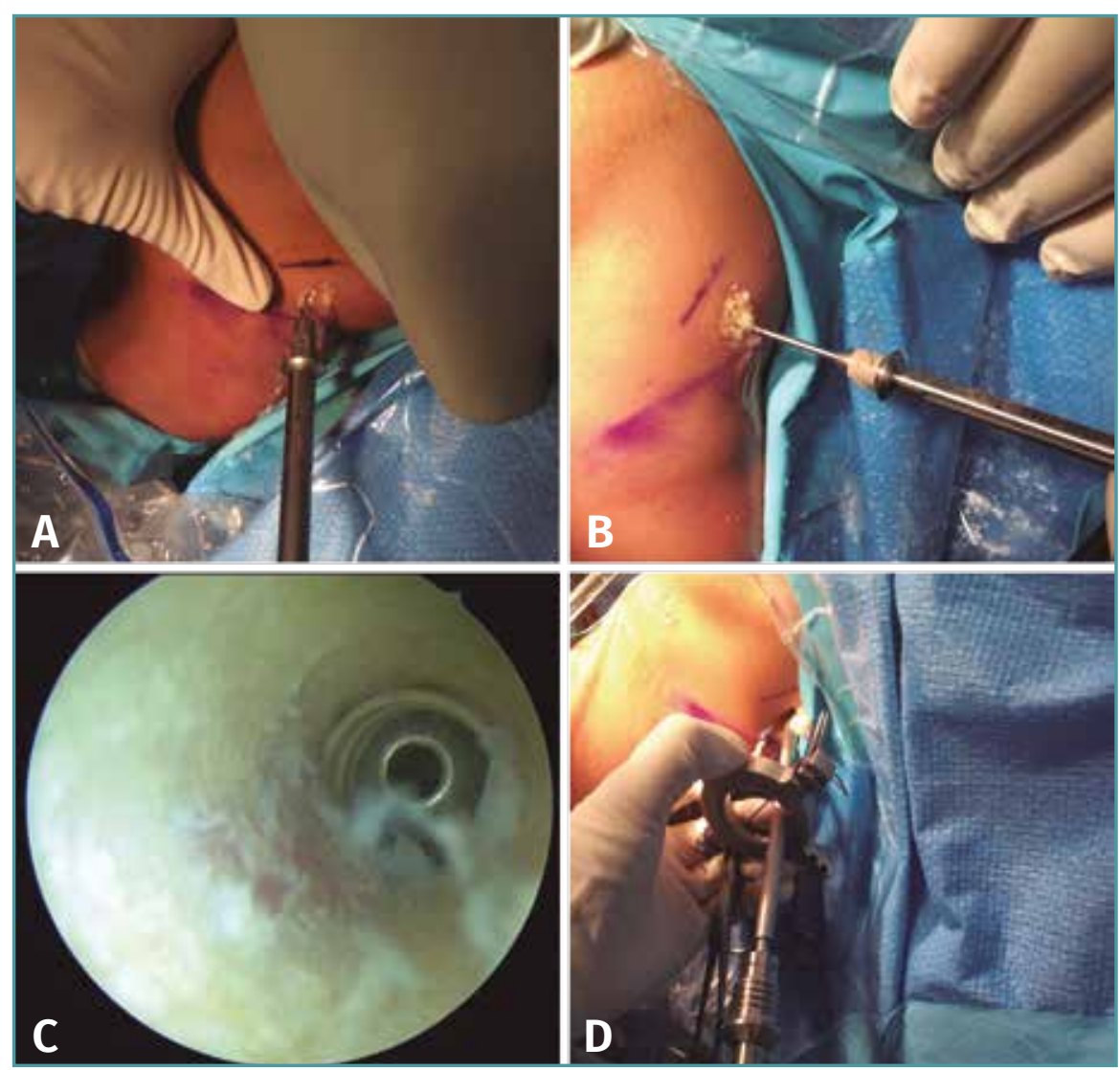

Figura 6. Tensado y fijación de la plastia. A: brocado para la camisa del tornillo interferencial (ShieldLoc Sleeve ${ }^{\circledR}$ ); B y C: inserción del ShieldLoc Sleeve ${ }^{\circledR}$ y comprobación de su correcta colocación; D: tensado de la plastia y fijación con tornillo (ShieldLoc Screw ${ }^{\circledR}$ ).
Los túneles utilizados han sido de $8 \mathrm{~mm}$ en 11 casos y $7 \mathrm{~mm}$ en 4 , con 1 caso de $6 \mathrm{~mm}$ y otro de $9 \mathrm{~mm}$ (Tabla 1). El tiempo transcurrido desde el diagnóstico de la lesión hasta la cirugía ha sido variable, entre los 2 y los 18 meses, con una media de 7,8 meses.

Se han descrito lesiones asociadas en 14 casos; 8 casos con lesiones en el menisco interno (MI): 4 casos presentaron rotura simple tratada con sutura en 2 casos y meniscectomía parcial en los 2 restantes; hubo 1 caso de rotura en asa de cubo y 1 caso de desinserción meniscal, ambos casos tratados también mediante sutura. Se registraron $4 \mathrm{Ca}-$ sos de lesión del menisco externo (ME): 2 casos de asa de cubo candidatos a sutura y otra, asociada a condropatía, que requirió meniscectomía parcial. En 1 caso hubo una lesión combinada en menisco externo e interno, 2 casos de condropatía, sin olvidar los 4 casos sin ninguna lesión asociada en la exploración artroscópica (Tabla2).

Se han dado 2 casos de complicaciones tardías: 

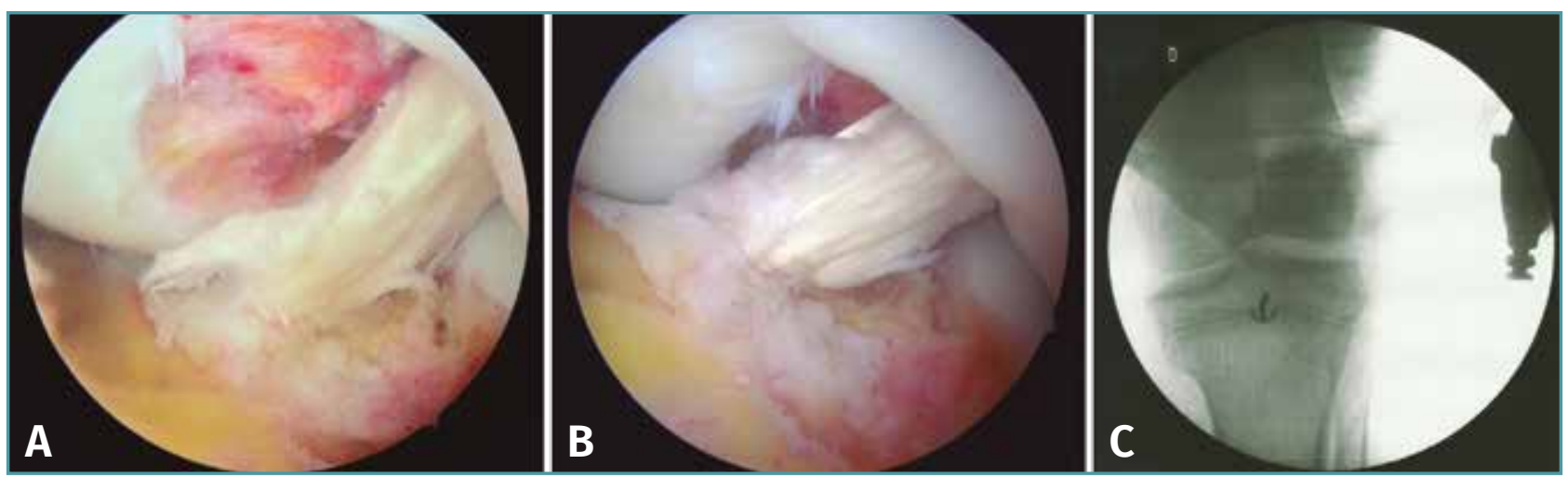

Figura 7. A y B: posición final de la plastia en flexión y extensión; C: vista radiológica final.

\begin{tabular}{|c|c|c|c|c|c|}
\hline Paciente & Edad & Sexo & Mecanismo & $\begin{array}{l}\text { Abstención } \\
\text { deportiva }\end{array}$ & Implicación social \\
\hline 1 & 12 & M & Cama elástica & 12 & $x$ \\
\hline 2 & 12 & $\mathrm{H}$ & Fútbol & 3 & Bajo rendimiento académico \\
\hline 3 & 12 & $\mathrm{H}$ & Fútbol & 3 & $\begin{array}{l}\text { Bajo rendimiento } \\
\text { académico. Obesidad }\end{array}$ \\
\hline 4 & 10 & $\mathrm{H}$ & Esquí & 3 & $x$ \\
\hline 5 & 9 & M & Traumatismo & 10 & $x$ \\
\hline 6 & 12 & $\mathrm{H}$ & Fútbol & 10 & Bajo rendimiento académico \\
\hline 7 & 12 & $\mathrm{H}$ & Fútbol & 10 & Bajo rendimiento académico \\
\hline 8 & 13 & $\mathrm{H}$ & Fútbol & 8 & Bajo rendimiento académico \\
\hline 9 & 14 & $\mathrm{H}$ & Baloncesto & 2 & Aislamiento ocasional \\
\hline 10 & 14 & M & Rugby & 3 & Aislamiento ocasional \\
\hline 11 & 14 & $\mathrm{H}$ & Fútbol & 3 & Aislamiento ocasional \\
\hline 12 & 15 & $\mathrm{H}$ & Fútbol & 2 & Aislamiento ocasional \\
\hline 13 & 13 & $M$ & Traumatismo & $x$ & $\begin{array}{l}\text { Aislamiento ocasional por } \\
\text { miedo a inestabilidad }\end{array}$ \\
\hline 14 & 12 & $\mathrm{H}$ & Fútbol & 6 & Aislamiento ocasional \\
\hline 15 & 10 & M & Esquí & 6 & $x$ \\
\hline 16 & 13 & $\mathrm{H}$ & Fútbol & $x$ & $x$ \\
\hline 17 & 12 & $\mathrm{H}$ & Fútbol & 3 & $x$ \\
\hline 18 & 11 & $\mathrm{H}$ & Fútbol & $x$ & Hiperactividad \\
\hline
\end{tabular}

e hiperactividad (TDAH) y otro caso con flexo secundario a estrechamiento intercondíleo severo que se solucionó tras una condiloplastia (Tabla 2).

La reinserción deportiva de estos pacientes se ha hecho entre los 4 y los 14 meses postoperatorios (media: 9 meses).

El periodo de inactividad ha tenido consecuencias sociales en 12 pacientes: el aislamiento ocasional por no participar en la actividad de su círculo habitual fue lo más frecuente, con un total de 6 casos; después, 5 casos con bajo rendimiento académico y 1 caso de obesidad secundaria a la inactividad (Tabla 3).

En la valoración del resultado final el $100 \%$ de los casos tiene ausencia de dolor. Dos pacientes refieren sensación de inestabilidad, uno de ellos presenta Lachman y pivot shift positivos, mientras que el restante solo tiene positividad para el Lachman. Un paciente presenta pivot shift positi-

uno de ellos con una rerrotura meniscal en el contexto de un trastorno de déficit de atención vo con Lachman negativo y ausencia de sensación de inestabilidad. Ambos fueron tratados por 
Tabla 2. Detalles quirúrgicos

\begin{tabular}{|c|c|c|c|c|c|c|}
\hline Paciente & $\begin{array}{l}\text { Tiempo hasta } \\
\text { cirugía }\end{array}$ & $\begin{array}{l}\text { Lesiones } \\
\text { asociadas }\end{array}$ & Injerto & Túnel & Otros & Complicaciones \\
\hline 1 & 11 & Rotura MI & Auto (ST-RI) & 8 & Sutura (FastFix ${ }^{\circledR}$ ) & No \\
\hline 2 & 8 & Rotura MI & Auto (ST-RI) & 7 & Meniscectomía parcial & No \\
\hline 3 & 15 & Rotura MI & Auto (ST-RI) & 8 & Meniscectomía parcial & No \\
\hline 4 & 3 & $x$ & Auto (ST-RI) & 8 & $x$ & No \\
\hline 5 & 15 & $x$ & Auto (ST-RI) & 6 & $x$ & No \\
\hline 6 & 12 & Rotura MI y ME & Auto (ST-RI) & 9 & Meniscectomía parcial & No \\
\hline 7 & 18 & Condropatía & Auto (ST-RI) & 8 & $x$ & No \\
\hline 8 & 12 & Asa de cubo ME & Banco (ST) & 8 & Sutura (CrossFix $\left.{ }^{\circledR}\right)$ & Flexo \\
\hline 9 & 2 & Asa de cubo MI & Banco (ST) & 8 & Sutura & No \\
\hline 10 & 3 & $\begin{array}{l}\text { Rotura ME + } \\
\text { condropatía }\end{array}$ & Banco (ST) & 8 & Sutura & No \\
\hline 11 & 3 & Asa cubo ME & Banco (ST) & 8 & Sutura & No \\
\hline 12 & 3 & $\begin{array}{l}\text { Rotura ME + } \\
\text { condropatía }\end{array}$ & Banco (ST) & 8 & Meniscectomía parcial & No \\
\hline 13 & 6 & Condropatía & Banco (ST) & 7 & $x$ & No \\
\hline 14 & 10 & Rotura MI & Banco (ST) & 7 & Sutura (CrossFix $\left.{ }^{\circledR}\right)$ & No \\
\hline 15 & 11 & $x$ & Banco (ST) & 7 & $x$ & No \\
\hline 16 & 2 & Lesión condral & Auto (ST-RI) & 8 & 11 m evolución & No \\
\hline 17 & 5 & Desinserción MI & Auto (ST-RI) & 8 & 16 m evolución & Nueva lesión meniscal \\
\hline 18 & 2 & $x$ & Auto (ST-RI) & 8 & $7 \mathrm{~m}$ evolución & No \\
\hline
\end{tabular}

El tiempo hasta la cirugía está indicado en meses

ME: menisco externo; MI: menisco interno; RI: recto interno; ST: semitendinoso

El diámetro del túnel está indicado en milimetros

el mismo equipo quirúrgico y no presentan clara repercusión funcional.

El balance articular es completo en 15 pacientes. Tres casos presentan una limitación leve: 2 casos con flexo $5^{\circ}$ y 1 caso con flexo $6^{\circ}$ (corresponde al paciente al que se le realizó la condiloplastia). Todos los casos refieren hacer vida normal sin limitación. Al realizar el KT1000, 15 pacientes no muestran diferencias en la laxitud, 2 casos presentan mayor laxitud que la contralateral y 1 caso mayor rigidez (Tabla 3). Solo uno de los casos de mayor laxitud corresponde con un paciente que refiere sensación de inestabilidad.

En esta serie no parece haber relación entre los hallazgos intraoperatorios (lesiones asociadas), el tiempo de inactividad o presentar mejor o peor evolución. No obstante, la potencia de nuestro estudio es demasiado baja para obtener conclusiones al respecto, aunque sí pueda resultar orientativo para nuevas revisiones futuras de la serie.

\section{Discusión}

\section{Elección de la técnica}

Las indicaciones quirúrgicas en la cirugía de reparación del LCA en la población infantil están consensuadas. Las opciones de tratamiento han de adecuarse a la edad esquelética y el grado de madurez con el fin de minimizar alteraciones en el crecimiento de la extremidad: banda iliotibial 
para Tanner I-II, transfisaria para pacientes más mayores con Tanner IV-V y la TIE de Anderson para pacientes Tanner I-||| $\left.\right|^{(3,5,6)}$.

La técnica más extendida y por la que hemos optado en este trabajo ha sido TIE para todos los pacientes esqueléticamente inmaduros ${ }^{(3)}$ por los peores resultados documentados para las técnicas extraarticulares ${ }^{(4)}$. El hecho de que la mayoría de las lesiones se produzcan a los 6-12 meses de la madurez esquelética(4) y los buenos resultados presentados por Anderson en 2003 en cuanto a función y deformidad ${ }^{(11)}$ hacen de la TIE la técnica de elección en pacientes jóvenes con un estadio madurativo Tanner I-III.

Nuestra experiencia con TIE es congruente con los resultados descritos por su autor original ${ }^{(11)}$. Se trata de una técnica que, correctamente realizada, aporta 2 ventajas principales:

1. Al preservar la fisis se minimiza el riesgo de lesión.

2. Permite colocar la plastia en una posición más horizontal que otras técnicas, aportando mayor estabilidad rotacional. En la cohorte presentada, los resultados de estabilidad a 1 año de seguimiento son satisfactorios.

\section{Repercusión social}

Las consecuencias sociales de la lesión del LCA en pacientes pediátricos están muy escasamente consideradas en la bibliografía. Aunque en muchas ocasiones se puede llevar a cabo vida normal con una rotura completa de LCA modificando las rutinas diarias, en el paciente pediátrico se recomienda tratamiento quirúrgico con mucha frecuencia $^{(2)}$. Estos pacientes tienen sus propias motivaciones( ${ }^{(6)}$, siguen siendo activos a pesar de la inestabilidad recurrente y no suelen colaborar en aspectos clínicos diseñados para adultos como puede ser simplemente la exploración física o los protocolos de readaptación o rehabilitación $n^{(3,4)}$. En nuestra cohorte se hace patente en la variabilidad en los tiempos de reinserción deportiva y que hay muchos pacientes que la inician antes de lo deseado en los protocolos. Las características de esta población no solo hacen, en muchas ocasiones, inefectivo el tratamiento conservador, sino que este efecto se ve magnificado por otros aspectos de su entorno en forma de repercusión social. En este trabajo se ha podido ver que, de
18 pacientes diagnosticados e intervenidos, 13 de ellos han iniciado su actividad deportiva antes de cumplir el año postoperatorio y, de ellos, 4 lo han hecho antes de los 9 meses. El caso especial del paciente con TDAH ilustra bien esta situación, porque no ha sido posible determinar cuándo comenzó a hacer actividad física al máximo nivel (Tabla 3). Estos hallazgos pueden tener relación con el hecho de que, de estos 18 pacientes, 6 de ellos estuvieron en situación de aislamiento social ocasional de su círculo habitual por no poder participar de forma activa y 5 de ellos tuvieron repercusión académica. Un total de 11 pacientes de 18 totales en los que las consecuencias del cambio y/o la detención de la actividad física y social habitual han tenido consecuencias negativas más allá del proceso médico mientras este se ha solucionado. No solo es el paciente quien sufre estas consecuencias sociales. En la actualidad el núcleo familiar participa de este tipo de actividades: padres y hermanos comparten aficiones y participan del mismo círculo social en el tiempo libre. El hecho de que el niño detenga su participación provoca a su vez que su familia lo haga.

Creemos que este hecho contribuye a la peor colaboración de los pacientes en edad infantil a los protocolos para tratamiento conservador, que también es causa de la alta incidencia de lesiones asociadas documentadas en el acto quirúrgico (14 pacientes de 18 totales) y de la cantidad de pacientes que inician la actividad deportiva antes de lo deseado (9 de forma temprana entre los 9 y los 12 meses y 4 de forma precoz antes de los 9 meses). Por esto defendemos el tratamiento quirúrgico para la estabilidad la rodilla en estos pacientes.

\section{Lesiones asociadas}

Los resultados previos en pacientes pediátricos con lesión del LCA han sido decepcionantes debido al diagnóstico tardío, la reticencia a la intervención quirúrgica y a la alta incidencia de lesiones concomitantes (50-65\%) $)^{(1)}$. Anderson insiste en que retrasos en la reconstrucción llevan a lesiones condrales y meniscales pero también a otras más severas interarticulares ${ }^{(12)}$ porque estos pacientes ni adaptan ni descienden la intensidad de su actividad física a pesar de los episodios recurrentes de inestabilidad. En nuestra serie se ha hallado un total de 12 pacientes con lesiones 
meniscales aisladas, 2 pacientes con condropatía y lesión meniscal conjuntamente y 2 pacientes con condropatía aislada. Del total de 14 pacientes con lesión meniscal, 4 requirieron meniscectomía parcial. Cabe señalar que en la población pediátrica la primera intención es la reparación meniscal, dejando la meniscectomía parcial como última opción dado el pronóstico degenerativo que conlleva su realización.

En la revisión sistemática sobre el momento óptimo en el que realizar la cirugía, el equipo de Andernord no encuentra diferencias en los resultados comparando intervenciones precoces y tardías cuando éstas se encuentren dentro del primer año desde el diagnóstico de la lesión en aquellos estudios con menor tiempo de seguimiento. Unas diferencias que sí se hacen evidentes con seguimientos a partir de 5 años desde la intervención ${ }^{(13)}$. Está claro que a mayor tiempo de evolución con inestabilidad, más riesgo de generar lesiones asociadas irreparables $^{(12)}$, algo que apoya nuestra incidencia del 77,8\% de lesiones asociadas, que es otra razón a favor del tratamiento quirúrgico porque los niños tienen mayor tasa de curación (78-84\%) tras el acto de reparación ${ }^{(5)}$. No obstante, Crawford et al. han publicado un trabajo en el que no encuentran diferencias en las lesiones intraarticulares entre aquellos que se intervienen de forma más temprana y más tardía ${ }^{(14)}$

\section{Complicaciones}

En este trabajo se han presentado 2 casos con sensación de inestabilidad tras la ligamentoplastia. En ambos se ha utilizado autoinjerto, obtienen un kt30 < 3 al año pero muestran un test de Lachman positivo. En uno de ellos se realizó una sutura meniscal de menisco interno y en el restante no se hallaron lesiones asociadas. En este último, además, el test pivot shift fue también positivo. Otros 3 casos presentan cierto grado de rigidez articular en forma de déficit de $5-6^{\circ}$ de extensión. Uno de ellos es portador de autoinjerto, que corresponde a aquel sin lesión asociada; y los 2 restantes lo son de aloinjerto. Ambos requirieron sutura meniscal y obtuvieron un kt30 $<3$. Uno de estos 2 pacientes corresponde al caso de flexo estructural posquirúrgico, que resolvió tras una trocleoplastia y cuya causa podría ser la inexperiencia del cirujano, ya que se trató de su primer caso, junto con una probable alteración biológica local. También hubo un caso de nueva rotura meniscal en el contexto de TDAH, otra muestra de que estos pacientes suelen mantener el nivel previo de actividad (Tablas 2 y 3 ).

En los resultados de Anderson con TIE en pacientes Tanner I-III no se describen alteraciones en el crecimiento o problemas funcionales ${ }^{(11)}$. La incidencia de alteraciones del crecimiento local tras una ligamentoplastia de LCA se sitúa en torno al $1,8 \%{ }^{(15)}$, pero Collins et al. informan en su revisión sistemática de que estas alteraciones son más frecuentes, están poco documentadas y que eso interfiere en el estudio etiológico de las mismas $^{(9)}$. Las fisis se cierran alrededor de los 15,6 y 17,1 en niños y 15 y 16,9 años ${ }^{(3)}$. Una lesión de un 7-9\% del área de la fisis es suficiente para provocar una lesión de la fisis; la interposición del tejido blando (plastia) previene la formación del puente óseo, pero una tensión excesiva da lugar a deformidades angulares ${ }^{(4)}$. Por todo ello, se recomienda realizar una telemetría de miembros inferiores previa a la cirugía con el fin de documentar resultados posteriores ${ }^{(6)}$.

\section{Tipos de injerto}

Las opciones actuales de injerto son autoinjerto, tendón cuadricipital y aloinjerto, con mayor tendencia a utilizar tendón cuadricipital ${ }^{(7)}$. Sabemos que la tasa de rerrotura en pacientes pediátricos es alta, alrededor del 19\% ${ }^{(16,17)}$. Sin embargo, la cirugía de revisión más frecuente es por lesión meniscal, condral, retirada de implantes o rigidez articular ${ }^{(17)}$, lo que es congruente con nuestros resultados, ya que nuestras 2 revisiones han sido por rotura meniscal y flexo estructural, y no por rotura de la plastia.

Además, aunque está descrito mayor porcentaje de roturas de aloinjerto en comparación con autoinjerto, esas diferencias no tienen significación estadística ${ }^{(17-20)}$; muchas revisiones sistemáticas están hechas a partir de estudios observacionales como la de Wasserstein et al. ${ }^{(21)}$. Si parece haber consenso en que la tasa de rotura de la plastia en general en pacientes pediátricos es mayor (22); nos hemos planteado iniciar la comparación aleatoria entre ambos tipos de injerto en el estudio actual con el fin de mejorar el seguimiento y el volumen poblacional a medida que nuestra serie crezca ya que, además, no existe 
una clara contraindicación al uso de aloinjerto, cuyo uso puede disminuir los tiempos quirúrgicos y la iatrogenia en el sitio de extracción.

Con el seguimiento a 1 año de nuestra cohorte no hemos encontrado diferencias en los resultados según el tipo de injerto ni en complicaciones ni en resultados finales. Como se ha señalado en el párrafo anterior, es conveniente revisar nuestros resultados más adelante.

\section{Limitaciones}

Se trata de una serie de 18 casos de la que, estadisticamente, no se podrían sacar conclusiones ni realizar comparaciones por una potencia escasa. Sin embargo, teniendo en cuenta la incidencia de esta lesión y que los trabajos que incluyen una importante cantidad de casos son revisiones a partir de estudios observacionales o no significativos, es una serie de casos que creemos tiene una $n$ suficiente para describir nuestra problemática, aunque no para sacar conclusiones en el momento actual. El tiempo de seguimiento es relativamente corto y no nos permite hablar de complicaciones importantes como dismetrías o alteraciones angulares; no obstante, es más que suficiente para valorar la reinserción deportiva y los resultados objetivos mediante la aplicación de KT1000 y nos abre las puertas a conformar un estudio evolutivo y comparativo en el tiempo.

\section{Conclusiones}

La ligamentoplastia mediante la TIE en pacientes Tanner I-III previene el avance o la aparición de otras lesiones intraarticulares, obtiene buenos resultados funcionales y una reinserción deportiva completa y soluciona los problemas sociales secundarios pero importantes como el aislamiento de su entorno habitual o consecuencias académicas que, a su vez, son en parte la causa de que estos pacientes no sigan bien las pautas de tratamiento conservador.

\section{Responsabilidades éticas}

Conflicto de interés. Los autores declaran no tener ningún conflicto de interés.
Financiación. Este trabajo no ha sido financiado.

Protección de personas y animales. Los autores declaran que para esta investigación no se han realizado experimentos en seres humanos ni en animales.

Confidencialidad de los datos. Los autores declaran que han seguido los protocolos de su centro de trabajo sobre la publicación de datos de pacientes.

Derecho a la privacidad y consentimiento informado. Los autores declaran que en este artículo no aparecen datos de pacientes.

\section{Bibliografía}

1. Siebold R, Seil R, Engebretsen L. ACL tear in kids: serious injury with high of osteoarthritis. Knee Surg Sports Traumatol Arthrosc. 2016:24:641-3.

2. Bigoni M, Gaddi D, Gorla M, Munegato D, Pungitore M. Arthroscopic anterior cruciate ligament repair for proximal anterior cruciate ligament tears in skeletally immature patients: surgical technique and preliminary results. Knee. 2017 Jan;24(1):40-8.

3. Dunn KL, Lam KC, Valovich McLeod TC. Early operative versus delayed or nonoperative treatment of anterior cruciate ligament injuries in pediatric patients. J Athl Train. 2016;51(5):425-7.

4. Larsen MW, Garrett WE Jr, Delee JC, Moorman CT 3rd. Surgical management of anterior cruciate ligament injuries in patients with open physes I Am Acad Orthop Surg. 2006 Dec;14(13):736-44.

5. Fabricant PD, Jones KJ, Delos D, Cordasco FA, Marx RG, Pearle AD. Reconstruction of the anterior cruciate ligament in the skeletally immature athlete: a review of current concepts: AAOS exhibit selection. J Bone Joint Surg Am. 2013 Mar 6;95(5):e28.

6. Lawrence J, Lee P, Kocher M. Anterior cruciate ligament reconstruction in the skeletally immature patient .En: Wiesel SW (ed.). Operative Techniques in Pediatric Orthopaedic Surgery. Philadelphia (PA): Lippincott Williams \& Wilkins; 2016. pp. 405-17.

7. Pennock A, Murphy MM, Wu M. Anterior cruciate ligament reconstruction in skeletally immature patients Curr Rev Musculoskelet Med. 2016;9:445-53.

8. Frobell RB, Roos EM, Roos HP, Ranstam J, Lohmander LS. A randomized trial of treatment for acute anterior cruciate ligament tears. N Engl J Med. 2010;22;363(4):331-42.

9. Collins MJ, Arns TA, Leroux T, Black A, Mascarenhas R, Bach BR Jr. Growth abnormalities following anterior cruciate ligament reconstruction in the skeletally im- 
mature patient: a systematic review. Arthroscopy. 2016 Aug;32(8):1714-2.

10. Anderson AF, Anderson CN. Transepiphyseal anterior cruciate ligament reconstruction in pediatric patients: surgical technique. Sports Health. 2009 Jan;1(1):76-80.

11. Anderson AF. Transepiphyseal replacement of the anterior cruciate ligament in skeletally immature patients. J Bone Joint Surg Am. 2003;85:1255-63.

12. Anderson AF, Anderson CN. Correlation of meniscal and articular cartiage injuries in children and adolescents with timing of anterior cruciate ligament reconstruction. AM J Sports Med. 2014;43(2):265-81.

13. Andernord D, Karlsson J, Musahl V, Bhandari M, Fu FH, Samuerlsson K. Timing of surgery of the anterior cruciate ligament. Arthroscopy. 2013;29(11):1863-71.

14. Crawford EA, Young LJ, Bedi A, Wojtys EM. The Effects of Delays in Diagnosis and Surgical Reconstruction of ACL Tears in Skeletally Immature Individuals on Subsequent Meniscal and Chondral Injury. J Pediatr Orthop. 2017 Feb 23. [Epub ahead of print].

15. Makani A, Franklin CC, Kanj WW, Wells L. All-epiphyseal anterior cruciate ligament reconstruction using fluoroscopy imaging J Pediatr Orthop B. 2013 Sep;22(5):445-9.

16. Nelson IR, Chen J, Love R, Davis BR, Maletis GB, Funahashi TT. A comparison of revision and re-rupture rates of $A C L$ reconstruction between autografts and allografts in the skeletally immature. Knee Surg Sports Traumatol Arthrosc. 2016;24:773-9.
17. Engelman GH, Carry PM, Hitt KG, Polousky JD, Vidal AF. Comparison of allograft versus autograft anterior cruciate ligament reconstruction graft survival in an active adolescent cohort. Am J Sports Med. 2014;42:2311-8.

18. Larson CM, Heikes CS, Ellingson Cl, Wulf CA, Giveans MR, Stone RM, Bedi A. Alograft and autograft transphyseal anterior cruciate ligament reconstruction in skeletally immature patients: outcomes and complications. Arthroscopy. 2016 May;32(5):860-7.

19. Kaeding CC, Aros B, Pedroza A, Pifel E, Amendola A, Andrish JT, et al. Allograft versus autograft anterior cruciate ligament reconstruction predictors of failure from a MOON prospective longitudinal cohort. Sports Health. 2011 Jan;3(1):73-81.

20. Nelson IR, Chen J, Love R, Davis BR, Maletis GB, Funahashi TT. A comparison of revision and re rupture rates of $\mathrm{ACL}$ reconstruction between autografts and allografts in the skeletally immature. Knee Surg Sports Traumatol Arthrosc. 2016 Mar;24(3):773-9.

21. Wasserstein D, Sheth U, Cabrera A, Spindler KP. A Systematic Review of Failed Anterior Cruciate Ligament Reconstruction With Autograft Compared With Allograft in Young Patients. Sports Health. 2015 May;7(3):207-16.

22. Dekker TJ, Godin JA, Dale KM, Garrett WE, Taylor DC, Riboh JC. Return to Sport After Pediatric Anterior Cruciate Ligament Reconstruction and its Effect on Subsequent Anterior Cruciate Ligament Injury. J Bone Joint Surg Am. 2017 Jun 7;99(11):897-904. 